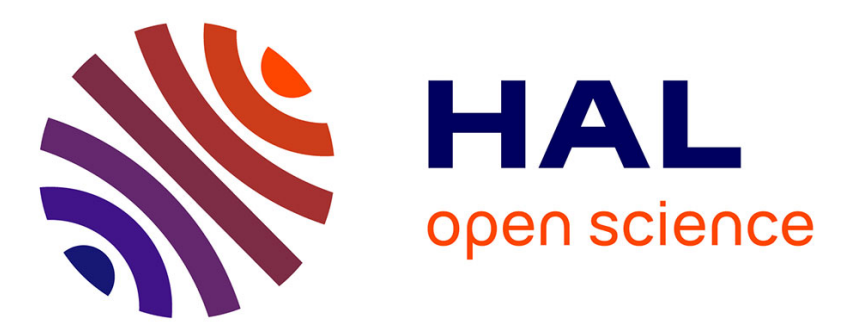

\title{
On induced-universal graphs for the class of bounded-degree graphs
}

Louis Esperet, Arnaud Labourel, Pascal Ochem

\section{To cite this version:}

Louis Esperet, Arnaud Labourel, Pascal Ochem. On induced-universal graphs for the class of boundeddegree graphs. Information Processing Letters, 2008, 108 (5), pp.255-260. 10.1016/j.ipl.2008.04.020 . hal-00368277

\section{HAL Id: hal-00368277 https://hal.science/hal-00368277}

Submitted on 15 Mar 2009

HAL is a multi-disciplinary open access archive for the deposit and dissemination of scientific research documents, whether they are published or not. The documents may come from teaching and research institutions in France or abroad, or from public or private research centers.
L'archive ouverte pluridisciplinaire HAL, est destinée au dépôt et à la diffusion de documents scientifiques de niveau recherche, publiés ou non, émanant des établissements d'enseignement et de recherche français ou étrangers, des laboratoires publics ou privés. 


\section{On induced-universal graphs for the class of bounded-degree graphs}

Louis Esperet, Arnaud Labourel, and Pascal Ochem 



\title{
On induced-universal graphs for the class of bounded-degree graphs
}

\author{
Louis Esperet* Arnaud Labourel ${ }^{\dagger}$ \\ Pascal Ochem $\ddagger$ \\ LaBRI UMR CNRS 5800, Université Bordeaux I, \\ 33405 Talence Cedex \\ FRANCE.
}

March 5, 2007

\begin{abstract}
For a family $\mathcal{F}$ of graphs, a graph $U$ is said to be $\mathcal{F}$-induced-universal if every graph of $\mathcal{F}$ is an induced subgraph of $U$. We give a construction for an induced-universal graph for the family of graphs on $n$ vertices with degree at most $k$. For $k$ even, our induced-universal graph has $O\left(n^{k / 2}\right)$ vertices and for $k$ odd it has $O\left(n^{\lceil k / 2\rceil-1 / k} \log ^{2+2 / k} n\right)$ vertices. This construction improves the main result of [9] by a multiplicative constant factor for even case and by almost a multiplicative $n^{1 / k}$ factor for odd case. We also construct induced-universal graphs for the class of oriented graphs with bounded incoming and outgoing degree, slightly improving another result of [9].
\end{abstract}

\section{Introduction}

All graphs are assumed to be without loops or multiples edges. For a graph $G$ we denote by $V(G)$ its vertex set and by $E(G)$ its edge or arc set. Our terminology is standard and any undefined term can be found in standard theory books [12].

For a finite family $\mathcal{F}$ of graphs, a graph $U$ is said to be $\mathcal{F}$-universal if every graph in $\mathcal{F}$ is a subgraph of $U$. For instance, if we denote by $\mathcal{F}_{n}$ the family of all graphs with at most $n$ vertices, then the complete graph $K_{n}$ is $\mathcal{F}_{n}$-universal. The problem of finding universal graphs was originally motivated by circuit design for computer chips [5]. The design cost of circuit is very expensive, whereas making many copies of the same circuit is rather inexpensive. This encouraged most of the chip manufacturers to make their circuits configurable.

\footnotetext{
*esperet@labri.fr

${ }^{\dagger}$ labourel@labri.fr

${ }^{\ddagger}$ ochem@labri.fr
} 
If we represent circuits by graphs, finding a configurable circuit may be viewed as finding a universal graph. These applications motivated the study of universal graphs for various families of graphs of $n$ vertices, including forests [11], bounded-degree forests [3, 4], and bounded-degree graphs [1].

The notion of induced-universal graph can be similarly defined. For a family $\mathcal{F}$ of graphs, a graph $U$ is $\mathcal{F}$-induced-universal if every graph in $\mathcal{F}$ is an induced subgraph of $U$. The family of all graphs on $n$ vertices was considered by Moon [14], while Chung considered trees, planar graphs, and graphs with bounded arboricity [10].

The problem of finding a small induced-universal graph is strongly related to a notion of distributed data structure known as adjacency labeling scheme. An adjacency labeling scheme for a family $\mathcal{F}$ of graphs consists in a labeling function that assigns labels to the vertices of any graph of $\mathcal{F}$ such that the adjacency can be decided between any two vertices by only looking at their labels. The problem of finding an adjacency labeling scheme with small labels was introduced by Breuer [7, 8]. Kannan, Naor and Rudich [13] established that there is an adjacency labeling scheme with labels of size $k(n)$ bits for the family $\mathcal{F}_{n}$ if and only if there exists an $\mathcal{F}_{n}$-induced-universal graphs with $2^{k(n)}$ vertices. This strong link between the two notions implies that any result on one notion has direct consequences on the other. For instance, the best known induced-universal graph for the class of forests is deduced from a labeling scheme [2].

In this paper, we focus on induced-universal graphs for bounded-degree graphs. We construct an induced-universal graph for the family $\mathcal{F}_{k, n}$ of graphs on $n$ vertices with degree at most $k$. For $k$ even, our induced-universal graph has $O\left(n^{k / 2}\right)$ vertices and for $k$ odd our induced-universal graph has $O\left(n^{\lceil k / 2\rceil-1 / k} \log ^{2+2 / k} n\right)$ vertices. Our result for graphs with maximum degree $k \equiv 0(\bmod 2)$ is deduced from a construction similar to that of [9] but with an improvement of the base graph of the construction (Section 3). Our result for graphs with maximum degree $k \equiv 1(\bmod 2)$ is deduced from a recent result of Alon and Capalbo [1] on universal graphs for bounded-degree graphs, combined with a construction of [10] that gives an interesting connection between induceduniversal graphs and universal graphs (Section 4). The best known lower bound for the number of vertices of an $\mathcal{F}_{k, n}$-induced-universal graph is $\Omega\left(n^{k / 2}\right)$ [9]. So, our result for $k$ even is tight up to a multiplicative constant and our result for $k$ odd is equal to $O\left(n^{1 / 2-1 / k} \log ^{2+2 / k} n\right)$ times the lower bound. We also give a generalization of our result for oriented graphs (Section 5). In Section 6 , we give a construction of an induced-universal graph for all orientations of the graphs of a family $\mathcal{F}$, only using a specific $\mathcal{F}$-induced-universal graph. We conclude the paper with some open problems (Section 7). 


\section{A small induced-universal graph for graphs with degree at most two}

Our main concern is to find an $\mathcal{F}_{k, n}$-induced-universal graphs for each $k$. We first investigate the case $k=2$.

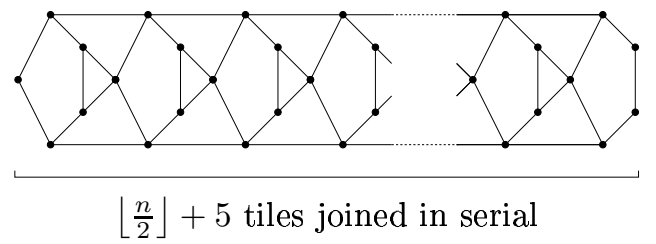

Figure 1: The $\mathcal{F}_{2, n}$-induced-universal graph $U_{n}$.

Lemma 1 The graph $U_{n}$ depicted in Figure 1 is an $\mathcal{F}_{2, n}$-induced-universal graph.

Proof. It is sufficient to prove that any graph $G \in \mathcal{F}_{2, n}$ is an induced subgraph of $U_{n}$, the graph depicted in Figure 1 . For $1 \leq i \leq n$, let $n_{i}$ be the number of connected components of $G$ with $i$ vertices. The degree of $G$ is bounded by 2 so $G$ contains $n_{1}$ isolated vertices, $n_{2}$ disjoint $K_{2}$ 's, and for $i \geq 3$, cycles or paths with $n_{i}$ vertices. We embed the connected components of $G$ into $U_{n}$ from left to right after having sort them by increasing size. The graph $U_{n}$ is made of cycles of size 5 called tiles that are joined in serial by 4 edges. Let us prove that we can embed all the connected components of $G$ in an induced way using at most $\left\lfloor\frac{n}{2}\right\rfloor+5$ tiles.

- The embedding of the stable set of size $n_{1}$, using $\left\lceil\frac{n_{1}}{2}\right\rceil+1$ tiles.

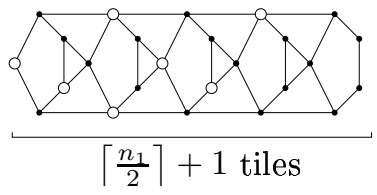

- The embedding of the $n_{2} K_{2}$ 's, using $n_{2}+1$ tiles.

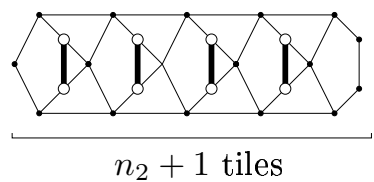

- The embedding of the $n_{3}$ connected components of size 3 , using $n_{3}+1$ tiles. 


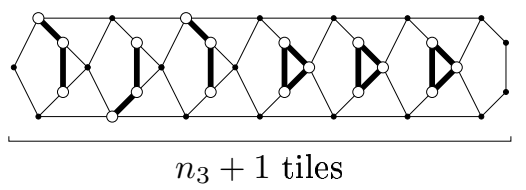

- The embedding of the $n_{4}$ connected components of size 4 , using $2 n_{4}+1$ tiles.

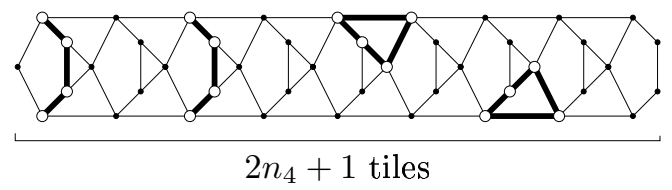

- The embedding of the $n_{5}$ connected components of size 5 , using $2 n_{5}$ tiles.

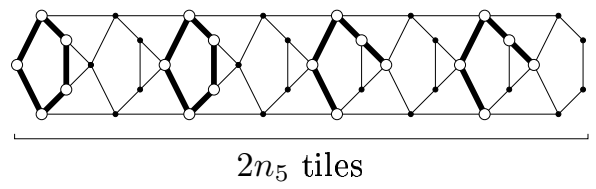

- For $k \geq 3$, the embedding of the $n_{2 k}$ connected components of size $2 k$, using $k n_{2 k}$ tiles.

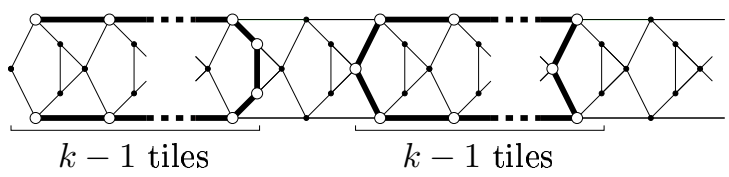

- For $k \geq 3$, the embedding of the $n_{2 k+1}$ connected components of size $2 k+1$, using $k n_{2 k+1}$ tiles.

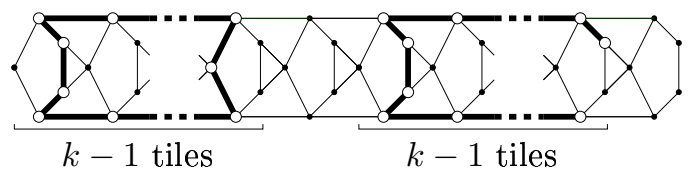

Observe that for each $i$ the embedding of connected components of size $i$ is induced. Moreover, at the end of the embedding of all connected components of size $i$, there is a tile in which no vertex of $G$ is embedded. So, there are no edges of $U_{n}$ between the embeddings of two connected components of different sizes. Hence, the embedding of $G$ into $U_{n}$ is induced. It remains to upper bound the number of tiles $l$ used by such an embedding. 


$$
\begin{aligned}
l & =\frac{n_{1}}{2}+2+n_{2}+1+n_{3}+1+2 n_{4}+1+2 n_{5}+\sum_{k=3}^{\lfloor n / 2\rfloor} 2 k n_{2 k}+\sum_{k=3}^{\lfloor n / 2\rfloor} 2 k n_{2 k+1} \\
& \leq 5+\sum_{i=1}^{n} i \frac{n_{i}}{2} \\
& \leq 5+\left\lfloor\frac{n}{2}\right\rfloor, \text { since } \sum_{i=1}^{n} i n_{i}=n \text { and the number of tiles is an integer. }
\end{aligned}
$$

A natural question is to investigate whether this construction is optimal. We now prove that it is optimal up to a constant multiplicative factor of approximatively $\frac{3}{2}$.

Claim 1 Every $\mathcal{F}_{2, n}$-induced-universal graph has at least $11\left\lfloor\frac{n}{6}\right\rfloor$ vertices.

Proof. Let $n \in \mathbb{N}$ be a multiple of 6 . Let $\mathcal{H}_{n}$ be the family containing the following three graphs:

- the stable set of $n$ vertices

- the disjoint union of $n / 2 K_{2}$, called induced matching

- the disjoint union of $n / 3 K_{3}$, called disjoint triangles

Note that all these three graphs have $n$ vertices and degree at most two. Let $U_{n}$ be an $\mathcal{H}_{n}$-induced-universal graph. Observe that the number of vertices in a maximal stable set in $n / 3$ disjoint triangles is $n / 3$ since each triangle cannot have more than one vertex in a stable set. So, the graph $U_{n}$ has a stable set of size $2 n / 3$ that is vertex disjoint of the embedding of the $n / 3$ triangles because otherwise it would not contain a stable set of size $n$. Two disjoint edges of the induced matching cannot both have endpoints embedded in vertices of the embedding of one triangle because otherwise there would be an edge between two endpoints of two edges of the induced matching. So, we cannot have more than $n / 3$ edges of the induced matching that have endpoints embedded in same vertices as the $n / 3$ triangles. The graph $U_{n}$ has a stable set of size $2 n / 3$ and an induced matching of $n / 2-n / 3=n / 6$ edges that are both vertex disjoint of the embeddings of the $n / 3$ triangles. A graph containing a stable set of size $2 n / 3$ and an induced matching of $n / 6$ edges must have $2 n / 3+n / 6=5 n / 6$ vertices since an edge cannot have more than one end in the stable set. Eventually, $U_{n}$ has at least $11 n / 6$ vertices and so any $\mathcal{F}_{2, n}$-induced-universal graph needs $11\lfloor n / 6\rfloor$ vertices because $\mathcal{H}_{6\lfloor n / 6\rfloor} \subseteq \mathcal{F}_{2, n}$. 


\section{Induced-universal graphs for graphs with even max- imum degree}

We now use our construction of an $\mathcal{F}_{2, n}$-induced-universal graph to construct an $\mathcal{F}_{k, n}$-induced-universal graph for $k$ even (the same method was already used in [9]).

Theorem 1 Let $k \geq 2$ be an even integer. There is an $\mathcal{F}_{k, n}$-induced-universal graph $U_{k, n}$ such that

$$
\left|V\left(U_{k, n}\right)\right|=(1+o(1))\left(\frac{5 n}{2}\right)^{k / 2} \text { and }\left|E\left(U_{k, n}\right)\right|=\left(\frac{9 k}{10}+o(1)\right)\left(\frac{5 n}{2}\right)^{k-1} .
$$

To prove this theorem, we first reduce the problem to the construction of an $\mathcal{F}_{2, n}$-induced-universal.

Theorem 2 (Chung [10]) Let $U$ be an $\mathcal{F}$-induced-universal graph. Suppose that $\mathcal{H}$ is a family of graphs such that each $H \in \mathcal{H}$ can be decomposed into $k$ edge-disjoint subgraphs each of which belongs to $\mathcal{F}$. Then there exists an $\mathcal{H}$ induced-universal graph $W$ where

$$
|V(W)|=|V(U)|^{k} \text { and }|E(W)|=k|V(U)|^{2 k-2}|E(U)| .
$$

Theorem 3 (Petersen [15]) Let $G$ be a k-regular graph, with $k$ even. G can be decomposed into $k / 2$ edge-disjoint graphs of degree at most 2.

Proof of Theorem 1. From Theorem 3, every graph $G \in F_{k, n}$ can be decomposed into $k / 2$ edge-disjoint graphs of degree at most 2. Using Lemma 1, we construct an $\mathcal{F}_{2, n}$-induced-universal graph $U_{n}$ with $\left|V\left(U_{n}\right)\right|=\frac{5}{2} n+O(1)$ and $\left|E\left(U_{n}\right)\right|=\frac{9}{2} n+O(1)$. Eventually, using Theorem 2, we obtain an $\mathcal{F}_{k, n}$-induceduniversal graph $U_{k, n}$ such that

$$
\begin{aligned}
\left|V\left(U_{k, n}\right)\right| & =|V(U)|^{k / 2} \\
& =\left(\frac{5}{2}\right)^{k / 2} n^{k / 2}+o\left(n^{k / 2}\right) \\
\left|E\left(U_{k, n}\right)\right| & =\frac{k}{2}|V(U)|^{k-2}|E(U)| \\
& =\frac{k}{2} \cdot \frac{9}{2}\left(\frac{5}{2}\right)^{k-2} n^{k-1}+o\left(n^{k-1}\right) .
\end{aligned}
$$

\section{Induced-universal graphs for graphs with odd max- imum degree}

To our best knowledge, there is no good result on edge decomposition for graphs belonging to $\mathcal{F}_{k, n}$ with $k$ odd. We cannot use any edge decomposition to reduce 
our problem to finding induced-universal graphs for simple families of graphs. Nevertheless, we can use $U_{k+1, n}$ as an $\mathcal{F}_{k, n}$-induced-universal graph since $\mathcal{F}_{k, n} \subset$ $\mathcal{F}_{k+1, n}$. The graph obtained is from a multiplicative factor of $O\left(n^{1 / 2}\right)$ of the best known lower bound for the number of vertices of $\mathcal{F}_{k, n}$-induced-universal graphs. We now show how to reduce the gap between lower and upper bounds with a construction deduced from universal graphs.

Theorem 4 Let $k \geq 3$ be an odd integer. There is an $\mathcal{F}_{k, n}$-induced-universal graph $U_{k, n}$ such that

$$
\left|V\left(U_{k, n}\right)\right|=c_{1}(k) n^{\lceil k / 2\rceil-1 / k} \log ^{2+2 / k} n \text { and }\left|E\left(U_{k, n}\right)\right|=c_{2}(k) n^{k-2 / k} \log ^{4+4 / k} n
$$

This result is deduced from the $\mathcal{F}_{k, n}$-universal graph obtained by Alon and Capalbo [1], using a result of Chung [10] that gives a construction of an $\mathcal{F}_{k, n^{-}}$ induced-universal graph from a $\mathcal{F}_{k, n}$-universal universal graph.

Theorem 5 (Alon and Capalbo [1]) For every $k \geq 3$, there is an $\mathcal{F}_{k, n^{-}}$ universal graph $H_{k, n}$ with $n$ vertices and at most $c(k) n^{2-2 / k} \log ^{4 / k} n$ edges, for some constant $c(k)$. Moreover $H_{k, n}$ is regular, i.e., each vertex of $V\left(H_{k, n}\right)$ has degree $c(k) n^{1-2 / k} \log ^{4 / k} n$.

Theorem 6 (Chung [10]) Let $A_{r}$ be a family of graphs with arboricity at most $r$. Let $G$ be an $A_{r}$-universal graph. There exists an $A_{r}$-induced-universal graph $H$ such that

$$
|V(H)|=\sum_{v \in V(G)}\left(d_{G}(v)+1\right)^{r} \text { and }|E(H)|=\sum_{u v \in E(G)}\left(d_{G}(u)+1\right)^{r} d_{G}(v)^{r-1} .
$$

Proof of Theorem 4. From Theorem 5, there exists an $\mathcal{F}_{k, n}$-universal graph $H_{k, n}$ with $n$ vertices having degree at most $c(k) n^{2-2 / k} \log ^{4 / k} n$. It is well-known that graphs of degree at $\operatorname{most} k \equiv 1(\bmod 2)$ have arboricity bounded by $\lceil k / 2\rceil$. Using Theorem 6 on $H_{k, n}$, we can construct an induced-universal graph $U_{k, n}$ for the family $\mathcal{F}_{k, n}=\mathcal{A}_{\lceil k / 2\rceil}$ such that

$$
\begin{aligned}
\left|V\left(U_{k, n}\right)\right| & =\sum_{v \in V\left(H_{k, n}\right)}\left(d_{H_{k, n}}(v)+1\right)^{\lceil k / 2\rceil} \\
\leq & \left|V\left(H_{k, n}\right)\right|\left(2 d_{H_{k, n}}\right)^{\lceil k / 2\rceil} \\
\leq & n\left(2 c(k) n^{1-2 / k} \log ^{4 / k} n\right)^{\lceil k / 2\rceil} \\
\leq & c_{1}(k) n^{\lceil k / 2\rceil-1 / k} \log ^{2+2 / k} n, \text { where } c_{1}(k)=(2 c(k))^{\lceil k / 2\rceil} \\
\left|E\left(U_{k, n}\right)\right| & =\sum_{u v \in E\left(H_{k, n}\right)}\left(d_{H_{k, n}}(u)+1\right)^{\lceil k / 2\rceil} d_{H_{k, n}}(v)^{\lceil k / 2\rceil-1} \\
\leq & \left|E\left(H_{k, n}\right)\right|\left(2 d_{H_{k, n}}\right)^{\lceil k / 2\rceil}\left(d_{H_{k, n}}\right)^{\lceil k / 2\rceil-1} \\
\leq & c(k) n^{2-2 / k} \log ^{4 / k} n\left(2 c(k) n^{1-2 / k} \log ^{4 / k} n\right)^{\lceil k / 2\rceil}\left(c(k) n^{1-2 / k} \log ^{4 / k} n\right)^{\lceil k / 2\rceil-1} \\
\leq & c_{2}(k) n^{k-2 / k} \log ^{4+4 / k}, \text { where } c_{2}(k)=(2 c(k))^{k+1} .
\end{aligned}
$$




\section{Induced-universal graphs for bounded-degree ori- ented graphs}

An orientation $\vec{G}$ of a graph $G$ consists in assigning to every edge of $G$ one of its two possible orientations. $\vec{G}$ is called an oriented graph and by definition, it cannot have loops nor opposite arcs. The construction of Section 3 can be easily generalized to the family $\mathcal{O}_{k, n}$ of all the orientations of the graphs of $\mathcal{F}_{2 k, n}$ having incoming and outgoing degree at most $k$. Indeed, both Theorems 2 and 3 have an oriented version, which can be used almost like in Section 3.

Theorem 7 There is an $\mathcal{O}_{k, n}$-induced-universal oriented graph $\overrightarrow{O_{k, n}}$ such that

$$
\left|V\left(\overrightarrow{O_{k, n}}\right)\right|=(1+o(1))(3 n)^{k} \text { and }\left|E\left(\overrightarrow{O_{k, n}}\right)\right|=(2+o(1))(3 n)^{2 k-1} .
$$

Proof. The construction of an induced-universal graph for $\mathcal{O}_{k, n}$ is almost the same as the construction for $\mathcal{F}_{2 k, n}$ presented in Section 3. The oriented version of Theorem 3 says that a graph with outgoing and incoming degree at most $k$ can be decomposed into $k$ edge-disjoint graphs having outgoing and incoming degree at most 1. Let $\overrightarrow{O_{n}}$ be the graph represented in Figure 2. So, if $\overrightarrow{O_{n}}$ is $\mathcal{O}_{1, n}$-induced-universal then, using Theorem 2, we can construct an $\mathcal{O}_{k, n^{-}}$ induced-universal graph $\overrightarrow{O_{k, n}}$ having $\left|V\left(\overrightarrow{O_{k, n}}\right)\right|=(1+o(1))(3 n)^{k}$ vertices and $\left|E\left(\overrightarrow{O_{k, n}}\right)\right|=(2+o(1))(3 n)^{2 k-1}$ edges. So, the only thing we need to prove is that $\overrightarrow{O_{n}}$ is $\mathcal{O}_{1, n}$-induced-universal.

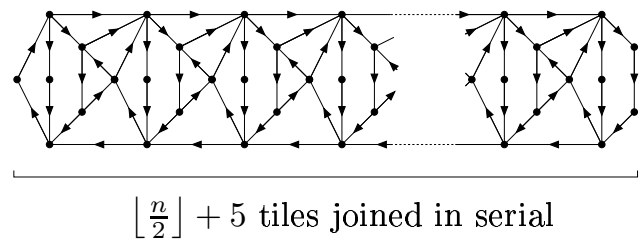

Figure 2: The $\mathcal{O}_{1, n}$-induced-universal graph $\overrightarrow{O_{n}}$.

Let $\vec{G}$ be any graph of $\mathcal{O}_{1, n}$. The connected components of $\vec{G}$ are either directed paths (oriented paths with exactly one sink and one source) or directed cycles (oriented cycles with no source). We embed $\vec{G}$ in $\overrightarrow{O_{n}}$ almost the same way we embedded graphs of $\mathcal{F}_{2, n}$ in $U_{n}$ in Section 2. The only differences are for the embeddings of connected components of size 3 or more that slighty differ from the non-oriented case. Indeed, we can embed stable sets and directed paths of size 2 using the same embedings as for the non-oriented case. We embed connected components of size 3 or more using the following scheme.

- The embedding of the $n_{3}$ connected components of size 3 , using $n_{3}+1$ tiles.

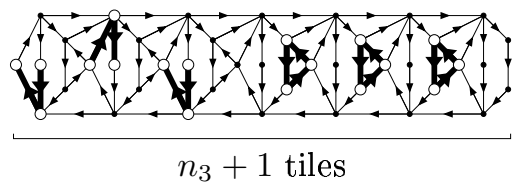


- The embedding of the $n_{4}$ connected components of size 4 , using $2 n_{4}+1$ tiles.

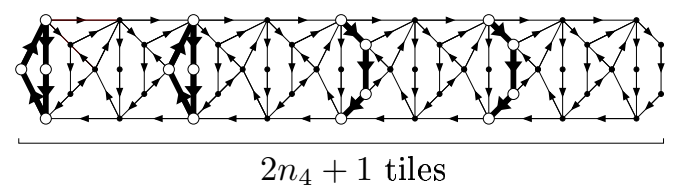

- The embedding of the $n_{5}$ connected components of size 5 , using $2 n_{5}+1$ tiles.

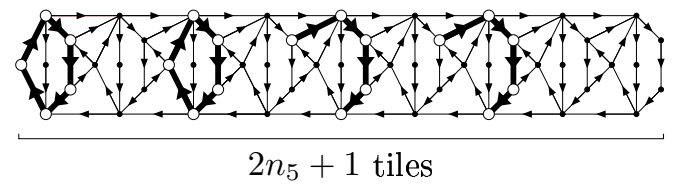

- For $k \geq 3$, the embedding of the $n_{2 k}$ connected components of size $2 k$, using $k n_{2 k}$ tiles.

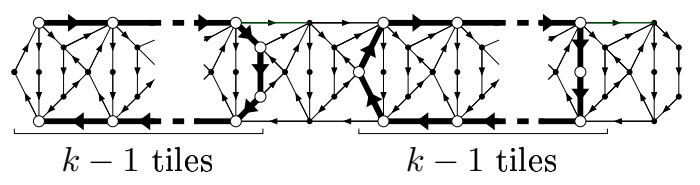

- For $k \geq 3$, the embedding of the $n_{2 k+1}$ connected components of size $2 k+1$, using $k n_{2 k+1}$ tiles.

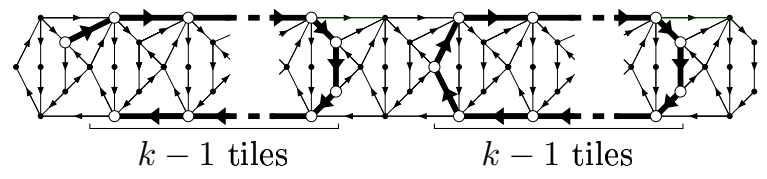

We use for embeddings exactly the same number of tiles as for the nonoriented case, so the graph $\overrightarrow{O_{n}}$ has the same number of tiles as the graph $U_{n}$ which had $\left\lfloor\frac{n}{2}\right\rfloor+5$ tiles.

\section{From induced-universal graphs to oriented induced- universal graphs}

In Section 5, we constructed an induced-universal graph for a family of orientations of graphs in $\mathcal{F}_{2, n}$ by orienting the edges and adding some vertices to the non-oriented induced-universal graph. Let $\mathcal{F}$ be a family of graph and $\overrightarrow{\mathcal{F}}$ be a family of orientations of graphs in $\mathcal{F}$. One may ask if it is always possible to construct an $\overrightarrow{\mathcal{F}}$-induced-universal graph $\vec{U}$ from an $\mathcal{F}$-induced-universal graph 
$U$. Given two graphs $G$ and $H$, a homomorphism from $G$ to $H$ is any mapping $f: V(G) \rightarrow V(H)$ satisfying $[x, y] \in E(G) \Rightarrow[f(x), f(y)] \in E(H)$. In fact, the construction is possible if there is a graph $\vec{H}$ into which each graph of $\overrightarrow{\mathcal{F}}$ has a homomorphism. In this case, the graph $\vec{H}$ is said to be an $\overrightarrow{\mathcal{F}}$-universal graph for homomorphism. For instance, the directed cycle of length three is a universal graph for homomorphism for the family of orientation of trees. The graph $\vec{U}$ can be obtained by making a special product of the two graphs $\vec{H}$ and $U$. The oriented tensor product $G \times \vec{H}$ of a non-oriented graph $G$ and an oriented graph $\vec{H}$ is defined to have vertex set $V(G \times \vec{H})=V(G) \times V(\vec{H})$ and arc set $E(G \times \vec{H})=\{[(x, u),(y, v)] \mid x y \in E(G)$ and $u v \in E(\vec{H})\}$.

Theorem 8 Let $U$ and $\vec{H}$ be two graphs. If $U$ is $\mathcal{F}$-induced-universal and $\vec{H}$ is $\overrightarrow{\mathcal{F}}$-universal for homomorphism then $U \times \vec{H}$ is $\overrightarrow{\mathcal{F}}$-induced-universal.

Proof. It suffices to show that we can embed an arbitrary graph $\vec{G} \in \overrightarrow{\mathcal{F}}$ as an induced subgraph of $U \times \vec{H}$. Let $v \in \vec{G}$. There is a homomorphism of $\vec{G}$ to $\vec{H}$ since $\vec{H}$ is $\overrightarrow{\mathcal{F}}$-universal for homomorphism. We denote by $h(v) \in V(\vec{H})$ the vertex into which $v$ is mapped. If we forget about the orientation, we can embed $\vec{G}$ into $U$ since $U$ is $\mathcal{F}$-induced-universal. Let denote by $u(v) \in V(U)$ the vertex into which $v$ is embedded. The embedding of $\vec{G}$ into $U \times \vec{H}$ consists in embedding each vertex $v$ of $G$ into the vertex $(u(v), h(v))$ of $U \times \vec{H}$. The embedding is correct in the sense that if there is an arc $[x, y]$ in $\vec{G}$ then there is an $\operatorname{arc}[(u(x), h(x)),(u(y), h(y))]$ in $U \times \vec{H}$. Indeed, there is an edge $[u(x), u(y)]$ in $U$ due to the non-oriented embedding of $\vec{G}$ into $U$ and an arc $[h(x), h(y)]$ in $\vec{H}$ due to the mapping of $\vec{G}$ into $\vec{H}$. Moreover, the embedding is induced. Indeed, if two vertices $x$ and $y$ of $G$ are not adjacent then $u(x)$ and $u(y)$ are not adjacent in $U$ because the non-oriented embedding of $\vec{G}$ into $U$ is induced. So, by construction, $(u(x), h(x))$ and $(u(y), h(y))$ are not adjacent in $U \times \vec{H}$.

The problem of finding an $\mathcal{F}$-universal graph for homomorphism is closely related to the oriented coloring of graphs of $\mathcal{F}$ and has been studied for various families of graphs. There is a universal graph for homomorphism with three vertices for trees, with 80 vertices for the family of planar graphs [6], with 7 vertices for 2-trees and outerplanar graphs [16], with 5 vertices for graph of degree at most 2 [16] and with 11 vertices for graphs of degree at most 3 [16]. This implies that for these families, we can construct an oriented induceduniversal graph for orientations of the family of graphs that have a number of vertices equal to a constant times the number of vertices of the induceduniversal graph. Observe that this construction is not optimal for $\mathcal{O}_{1, n}$ in the sense that the smallest $\mathcal{O}_{1, n}$-universal graph for homomorphism has exactly 5 vertices whereas our $\mathcal{O}_{1, n}$-induced-universal graph has less than five times the number of vertices of minimal $\mathcal{F}_{2, n}$-induced-universal. 


\section{Concluding remarks and open problems}

In Section 2, we proved that a minimal $\mathcal{F}_{2, n}$-induced-universal has at least $5 n / 2+O(1)$, and and at most $11 n / 6+O(1)$ vertices. The natural question that arises is whether it is possible to reduce the gap between $5 / 2$ and 11/6 for the multiplicative constant. This question seems to be quite difficult, even though graphs of $\mathcal{F}_{2, n}$ have a very simple structure. For $k$ odd, if we forget the polylogarithmic factor, there remains a multiplicative factor of $n^{1 / 2-1 / k}$ between the lower and the upper bound for the number of vertices in a minimal $\mathcal{F}_{k, n^{-}}$ induced-universal graph. An interesting problem would be to lower this factor, especially for large values of $k$. In our construction, for $k$ even, our $\mathcal{F}_{k, n}$-induceduniversal graph have maximum degree $4^{k / 2}$ depending only on $k$ whereas for $k$ odd, it has maximum degree $c_{2}(k) n^{k-1-2 / k} \log ^{4+4 / k} n$. Considering that for $k$ even our construction is almost tight whereas for $k$ odd it is not, we conjecture that $\mathcal{F}_{k, n}$-induced-universal graphs with minimal number of vertices and edges have degree only depending on $k$. In other words, we conjecture that there is a function $f(k)$ such that the existence of a $\mathcal{F}_{k, n}$-induced-universal graph $U_{k, n}$ implies that there exists another one with at most the same number of vertices, but with degree at most $f(k)$.

\section{References}

[1] N. Alon and M. Capalbo. Sparse universal graphs for bounded degree graphs. to appear.

[2] S. Alstrup and T. Rauhe. Small induced-universal graphs and compact implicit graph representations. In $43^{\text {rd }}$ Annual IEEE Symposium on Foundations of Computer Science (FOCS), pages 53-62. IEEE Computer Society Press, November 2002.

[3] S. Bhatt, F. Chung, F. Leighton, and A. Rosenberg. Optimal simulations of tree machines. In 27th IEEE Foundations of Computer Science, pages 274-282, Toronto, 1986.

[4] S. N. Bhatt, F. R. K. Chung, F. T. Leighton, and A. L. Rosenberg. Universal graphs for bounded-degree trees and planar graphs. SIAM J. Discret. Math., 2(2):145-155, 1989.

[5] S. N. Bhatt and C. E. Leiserson. How to assemble tree machines. F. Preparata, 1984.

[6] O. V. Borodin, A. V. Kostochka, J. Nešetřil, A. Raspaud, and E. Sopena. On universal graphs for planar oriented graphs of a given girth. Discrete Math., 188:73-85, 1998.

[7] M. A. Breuer. Coding the vertexes of a graph. IEEE Transactions on Information Theory, IT-12:148-153, 1966. 
[8] M. A. Breuer and J. Folkman. An unexpected result on coding the vertices of a graph. Journal of Mathematical Analysis and Applications, 20:583-600, 1967.

[9] S. Butler. Induced-universal graphs for graphs with bounded maximum degree. preprint.

[10] F. R. K. Chung. Universal graphs and induced-universal graphs. Journal of Graph Theory, 14:443-454, 1990.

[11] F. R. K. Chung and R. L. Graham. On universal graphs for spanning trees. J. London Math. Soc., 27:203-211, 1983.

[12] R. Diestel. Graph Theory, volume 173 of Graduate Texts in Mathematics. Springer-Verlag, Berlin, third edition edition, 2005.

[13] S. Kannan, M. Naor, and S. Rudich. Implicit representation of graphs. In $20^{\text {th }}$ Annual ACM Symposium on Theory of Computing (STOC), pages 334-343. ACM Press, May 1988.

[14] J. W. Moon. On minimal n-universal graphs. In Proc. Galasgow Math. Soc, volume 7, pages 32-33, 1965.

[15] J. Petersen. Die theorie der regulären graphs. Acta Math., 15:193-220, 1891.

[16] E. Sopena. The chromatic number of oriented graphs. J. Graph Theory, 25:191-205, 1997. 